\title{
Adipocyte-released insulin-like growth factor-1 is regulated by glucose and fatty acids and controls breast cancer cell growth in vitro
}

\author{
V. D'Esposito • F. Passaretti • A. Hammarstedt • \\ D. Liguoro • D. Terracciano • G. Molea • L. Canta • \\ C. Miele • U. Smith • F. Beguinot • P. Formisano \\ Received: 5 April 2012 /Accepted: 30 May 2012 /Published online: 15 July 2012 \\ (C) The Author(s) 2012. This article is published with open access at Springerlink.com
}

\begin{abstract}
Aims/hypothesis Type 2 diabetes and obesity are associated with increased risk of site-specific cancers. We have investigated whether metabolic alterations at the level of adiposederived differentiating cells may affect specific phenotypes of breast cancer cells.

Methods Growth profiles of breast cancer cell lines were evaluated in co-cultures with differentiated adipocytes or their precursor cells and upon treatment with adipocyte
\end{abstract}

Electronic supplementary material The online version of this article (doi:10.1007/s00125-012-2629-7) contains peer-reviewed but unedited supplementary material, which is available to authorised users.

V. D'Esposito · F. Passaretti • D. Terracciano · F. Beguinot •

P. Formisano $(\bowtie)$

Department of Cellular and Molecular Biology and Pathology,

Federico II University of Naples,

Via Pansini 5,

80131 Naples, Italy

e-mail: fpietro@unina.it

V. D'Esposito · D. Liguoro • C. Miele · F. Beguinot - P. Formisano Istituto di Endocrinologia ed Oncologia Sperimentale del C.N.R, Federico II University of Naples,

Naples, Italy

\section{F. Passaretti}

Department of Pharmaceutical and Biomedical Sciences, University of Salerno,

Salerno, Italy

\footnotetext{
A. Hammarstedt $\cdot$ U. Smith

Göteborg, Sweden

G. Molea $\cdot$ L. Canta

Department of Systematic Pathology,

Federico II University of Naples,

Naples, Italy
}

The Lundberg Laboratory for Diabetes Research,

Department of Molecular and Clinical Medicine/Diabetes,

The Sahlgrenska Academy, University of Göteborg, conditioned media. Production and release of cytokines and growth factors were assessed by real-time RT-PCR and multiplex-based ELISA assays.

Results Co-cultures with either differentiated mouse 3T3L1 or human mammary adipocytes increased viability of MCF-7 cells to a greater extent, when compared with their undifferentiated precursors. Adipocytes cultured in $25 \mathrm{mmol} / 1$ glucose were twofold more effective in promoting cell growth, compared with those grown in $5.5 \mathrm{mmol} / \mathrm{l}$ glucose, and activated mitogenic pathways in MCF-7 cells. Growth-promoting action was also enhanced when adipocytes were incubated in the presence of palmitate or oleate. Interestingly, 3T3-L1 and human adipocytes released higher amounts of keratinocyte-derived chemokine/IL-8, the protein 'regulated upon activation, normally Texpressed, and secreted' (RANTES), and IGF-1, compared with their precursor cells. Their levels were reduced upon incubation with low glucose and enhanced by fatty acids. Moreover, both undifferentiated cells and differentiated adipocytes from obese individuals displayed about twofold higher IGF-1 release and MCF-7 cell growth induction than lean individuals. Finally, inhibition of the IGF-1 pathway almost completely prevented the growth-promoting effect of adipocytes on breast cancer cells.

Conclusions/interpretation IGF-1 release by adipocytes is regulated by glucose and fatty acids and may contribute to the control of cancer cell growth in obese individuals.

Keywords Adipocytes · Breast cancer · IGF-1

$\begin{array}{ll}\text { Abbreviations } & \\ \text { Adipo 3T3-L1 } & \text { 3T3-L1 adipocytes } \\ \text { AU } & \text { Arbitrary units } \\ \text { bFGF } & \text { Basic fibroblast growth factor } \\ \text { CM } & \text { Conditioned medium } \\ \text { ER } & \text { Oestrogen (estrogen) receptor }\end{array}$




\begin{tabular}{|c|c|}
\hline ERK & Extracellular signal-regulated kinase \\
\hline G-CSF & Granulocyte-colony stimulating factor \\
\hline GM-CSF & $\begin{array}{l}\text { Granulocyte-macrophage colony } \\
\text { stimulating factor }\end{array}$ \\
\hline hAdipo & Human adipocyte \\
\hline HG & High glucose \\
\hline JAK & Janus kinase \\
\hline $\mathrm{KC}$ & Keratinocyte-derived chemokine \\
\hline LG & Low glucose \\
\hline MAPK & Mitogen-activated protein kinase \\
\hline MCP-1 & Monocyte chemoattractant protein 1 \\
\hline $\mathrm{MIP}-1 \alpha / \beta$ & $\begin{array}{l}\text { Macrophage inflammatory protein } \\
\text { 1-alpha/beta }\end{array}$ \\
\hline OLE & Oleate \\
\hline PAL & Palmitate \\
\hline PDGF & Platelet-derived growth factor \\
\hline PI3K & Phosphatidylinositol 3-kinase \\
\hline $\mathrm{PKB} / \mathrm{Akt}$ & Protein kinase B \\
\hline RANTES & $\begin{array}{l}\text { Regulated upon activation normally } \mathrm{T} \\
\text { expressed, and secreted }\end{array}$ \\
\hline STAT & $\begin{array}{l}\text { Signal transducer and activator of } \\
\text { transcription }\end{array}$ \\
\hline SVF & Stromal vascular fraction \\
\hline 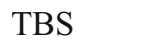 & Tris-buffered saline \\
\hline $\mathrm{VEC}$ & Vascular endothelial growth factor \\
\hline
\end{tabular}

\section{Introduction}

It has recently become clear that obesity and type 2 diabetes are associated with an increased frequency of many cancers $[1,2]$. For instance, increased adiposity is associated with an increased risk of several cancers [3]. Similarly, epidemiological data link type 2 diabetes with an increased incidence of multiple types of cancer, including breast, colorectal, hepatocellular, endometrial and pancreatic malignancies [4, 5]. Both obesity and diabetes can also lead to poorer treatment outcome and increase cancerrelated mortality rates [6-8].

The adipocyte may represent a candidate to integrate energy and nutrient metabolism with cancer cell growth by regulating cell functions through a complex network of endocrine, paracrine and autocrine signals [9-11]. Indeed, in addition to storing excess calories in the form of lipid, adipose tissue is an active endocrine organ that can have farreaching effects on the physiology of other tissues [12]. It has been shown that several hormones, growth factors and cytokines are produced or generated in white adipose tissue [13]. Recent interest has centred on the role of adipokines, chiefly leptin and adiponectin, and inflammatory cytokines [14]. However, all of the adipocyte factors may be envisioned as contributing factors for cancer onset or progression [15]. Moreover, adipocytes are largely represented in the microenvironment of several tumours, possibly providing a number of signals and resources to tumour cells.

The adipocyte represents one of the most abundant cell types surrounding breast cancer cells and may prove to be a key player in the stromal-ductal epithelial cell interactions within the mammary microenvironment [16]. In this regard, several in-vitro and in-vivo studies demonstrated that adipocytes can directly influence breast tumour growth [17].

Obesity has been consistently shown to increase the rate of breast cancer occurrence in postmenopausal women by $30-50 \%$ [11]. Studies of breast cancer mortality rates and survival illustrate that adiposity is associated both with poorer survival and increased likelihood of recurrence among those with the disease, regardless of menopausal status and after adjustment for disease stage and treatment. Very obese women $(B M I \geq 40.0)$ have breast cancer death rates that are three times higher than very lean $(\mathrm{BMI}<20.5)$ women $[3,18]$. Moreover, case-control and cohort studies showed that women with diabetes may have a $20 \%$ increased risk of breast cancer [19] and increased cancerrelated mortality rate [20].

Nevertheless, whether adipocyte-derived factors are directly involved in breast cancer onset or progression is still poorly understood [21]. The effect on tumour phenotype of metabolic alterations at the level of the adipose tissue is also unclear.

Here, we show that adipocytes may integrate inputs from the metabolic environment and promote growth of breast cancer cells. Indeed, both glucose and fatty acids enhance the ability of adipocytes to produce factors, including IL-8, RANTES (regulated upon activation, normally T expressed, and secreted) and IGF-1, involved in the control of cancer cell phenotypes. Finally, both stromal vascular fraction (SVF) cells and differentiated adipocytes from obese individuals release more IGF-1 than those from lean individuals, suggesting that obesity per se may enhance breast cancer cell growth.

\section{Methods}

Materials Media, sera and antibiotics for cell culture were from Lonza (Basel, Switzerland). Antibodies against phospho-Ser $_{473}$ protein kinase B (PKB/Akt1), forms of extracellular signal-regulated kinase (ERK), signal transducer and activator of transcription (STAT) 3 and actin were purchased from Santa Cruz Biotechnology (Santa Cruz, CA, USA). Phospho- $\mathrm{Thr}_{202} / \mathrm{Tyr}_{204}$ ERK and phospho- $\mathrm{Tyr}_{705}$ STAT3 antibodies were obtained from Cell Signaling Technology (Danvers, MA, USA). PKB/Akt antibody was from Millipore (Billerica, MA, USA). Anti-leptin antibodies were a generous gift from G. Matarese (CNR, Naples, Italy). Sodium dodecyl sulfate-polyacrylamide gel electrophoresis 
(SDS-PAGE) reagents were from Bio-Rad (Hercules, CA, USA). All the other chemicals were from Sigma-Aldrich (St Louis, MO, USA).

Cell cultures MCF-7 (ER $\alpha$ positive) and MDA-MB-231 (ER $\alpha$ negative) human breast cancer cells and 3T3-L1 mouse fibroblasts were cultured in DMEM supplemented with $10 \%$ fetal bovine serum (FBS) and $2 \mathrm{mmol} / 1$ glutamine, $100 \mathrm{IU} / \mathrm{ml}$ penicillin and $100 \mathrm{IU} / \mathrm{ml}$ streptomycin. Cultures were maintained in a humidified atmosphere of $95 \%$ air and $5 \% \mathrm{CO}_{2}$ at $37^{\circ} \mathrm{C}$. $3 \mathrm{~T} 3-\mathrm{L} 1$ differentiation was achieved as previously described [22]. Human adipose tissue samples were obtained from mammary adipose tissue and from abdominal subcutaneous adipose tissue biopsies. In detail, mammary adipose tissue specimens were obtained from women ( $n=12$; age 25-63 years; BMI 24.2-29) undergoing surgical mammary reduction. All women were otherwise healthy and free from metabolic or endocrine diseases. Abdominal adipose tissue was obtained from obese $(n=10$; age 45-65 years; BMI 30-35) and lean women ( $n=10$; age 32-60 years; BMI 20-25) by abdominal biopsy in the periumbilical region under local anaesthesia ( $2 \%$ lidocaine). Informed consent was obtained from every study participant before the surgical procedure. This procedure was approved by the ethical committee of the University of Naples. Adipose tissue was digested with collagenase and then adiposederived SVF cells were isolated and differentiated as previously reported [23]. Conditioned media were obtained by incubating the cells for $8 \mathrm{~h}$ with serum-free DMEM containing $0.25 \%$ BSA after two washes with PBS. After the incubation, medium was collected and centrifuged at $14,000 \mathrm{~g}$ to remove cellular debris and placed onto recipient cells for different times, as indicated, or analysed for cytokines and growth factor content, as described below. For co-cultures, $8 \times 10^{4} \mathrm{MCF}-7$ cells were seeded in the upper chamber of a transwell culture system ( $4 \mu \mathrm{m}$ pore size, Costar plates, Corning Life Sciences, Lowell, MA, USA) in a complete medium. The following day, the cells were incubated in serum-free DMEM 0.25\% BSA with or without adipocytes in the lower chamber. Sodium oleate and sodium palmitate were dissolved in $\mathrm{NaOH}$ at $100 \mathrm{mmol} / \mathrm{l}$ final concentration and conjugated with $10 \%$ fatty acid-free BSA (as a physiological carrier) at a molar ratio of $3: 1$ [24].

Cell proliferation and viability MCF-7 $\left(1 \times 10^{5}\right.$ cells/well $)$ and MDA-MB-231 $\left(1 \times 10^{5}\right.$ cells/well $)$ cells were seeded in six-well culture plates in a complete medium. The following day, the cells were starved in serum-free DMEM $0.25 \%$ BSA for $16 \mathrm{~h}$ and incubated with conditioned media obtained as described above for different times. Cell count was performed either by Bürker chamber and with the TC10 Automated Cell Counter (Bio-Rad) according to the manufacturer's protocol. Sulforhodamine assay or crystal violet staining were used for cell viability determination $[25,26]$.

Flow cytometry MCF-7 cells were kept in the presence or in the absence of serum or incubated with conditioned media, as indicated. Cell cycle phases were then assayed by cytometric analysis as previously described [27].

Cytokine and growth factor assay 3T3-L1 and human preadipocyte and adipocyte conditioned media were screened for the concentration of IL-1a, IL-1b, IL-2, IL-3, IL-4, IL-5, IL-6, IL-9, IL-10, IL-12 (p40), IL-12 (p70), IL-13, IL-17, eotaxin, granulocyte-colony stimulating factor (G-CSF), granulocyte-macrophage colony stimulating factor (GM$\mathrm{CSF}$ ), IFN- $\gamma$, keratinocyte-derived chemokine (KC)/IL-8, monocyte chemoattractant protein 1 (MCP-1), macrophage inflammatory protein 1-alpha/beta (MIP-1 $\alpha$, MIP-1 $\beta$ ), RANTES, TNF- $\alpha$, platelet-derived growth factor (PDGF), vascular endothelial growth factor (VEGF), basic fibroblast growth factor (bFGF) and IGF-1, using the Bio-Plex multiplex Mouse and Human Cytokine and Growth factor kits (Bio-Rad) according to the manufacturer's protocol.

Real-time RT-PCR analysis Total RNA was isolated from 3T3-L1 and human pre-adipocytes and adipocytes by using the Rneasy Kit (Qiagen Sciences, Germantown, MD, USA) according to the manufacturer's instruction. For real-time RT-PCR analysis, $1 \mu \mathrm{g}$ cell RNA was reverse transcribed using SuperScript II Reverse Transcriptase (Invitrogen, Carlsbad, CA, USA). PCRs were analysed using SYBR Green mix (Invitrogen). Reactions were performed using Platinum SYBR Green Quantitative PCR Super-UDG using an iCycler IQ multicolor Real-Time PCR Detection System (Bio-Rad). All reactions were performed in triplicate and $\beta$-actin was used as an internal standard. Primer sequences used are described in electronic supplementary material (ESM) Table 1.

Immunoblot procedure Total cell lysates were obtained and separated by SDS-PAGE as previously described [27].

Phosphoprotein assay Protein lysates were prepared by using Cell lysis kit (Bio-Rad) and the presence of phosphorylated Akt/PKB, ERK, STAT3 and IGF-1R was detected by Bio-Plex Phospho- Ser 473 Akt1/PKB, Phospho-Thr ${ }_{202} /$ Tyr $_{204}$ ERK, Phospho-Tyr ${ }_{705}$ STAT3 and Phospho-Tyr 1131 IGF-1R Assay kits and the Phosphoprotein Testing Reagent kit (Bio-Rad) according to the manufacturer's protocol. The total proteins for Akt1/PKB, ERK and STAT3 were tested using the Bio-Plex 3-plex assay kit (Bio-Rad).

Statistical analysis Data were analysed with Statview software (Abacus concepts) by one-factor analysis of variance. 
A $p$-value of less than 0.05 was considered statistically significant.

\section{Results}

Adipocytes promote breast cancer cell survival We first analysed the impact of adipocyte-secreted factors on breast cancer cell growth, in comparison with factors released by undifferentiated fibroblasts. Conditioned medium (CM) from 3T3-L1 adipocytes induced a time-dependent increase of cell growth, which was significantly more effective than that induced by CM from 3T3-L1 fibroblasts (Fig. 1a) and similar to that observed for cells incubated with $10 \%$ FBS-medium (Fig. 1a). Consistent results were obtained with the ER $\alpha$ negative MDA-MB-231 cells (Fig. 1b), suggesting that the effect was independent of oestrogen release. To investigate whether human adipocytes were as effective as murine cell lines in promoting breast cancer cell growth, we obtained adipose tissue from breast surgery. As shown in Fig. 1c, CM from adipocytes, either differentiated from SVF or directly isolated from mammary specimens, induced growth of MCF7 cells, by 1.5 fold and twofold, respectively, while $\mathrm{CM}$ from undifferentiated SVF cells had no significant effect. Similar data were also obtained with MDA-MB-231 cells (data not shown). Consistently, as assessed by sulforhodamine assay,
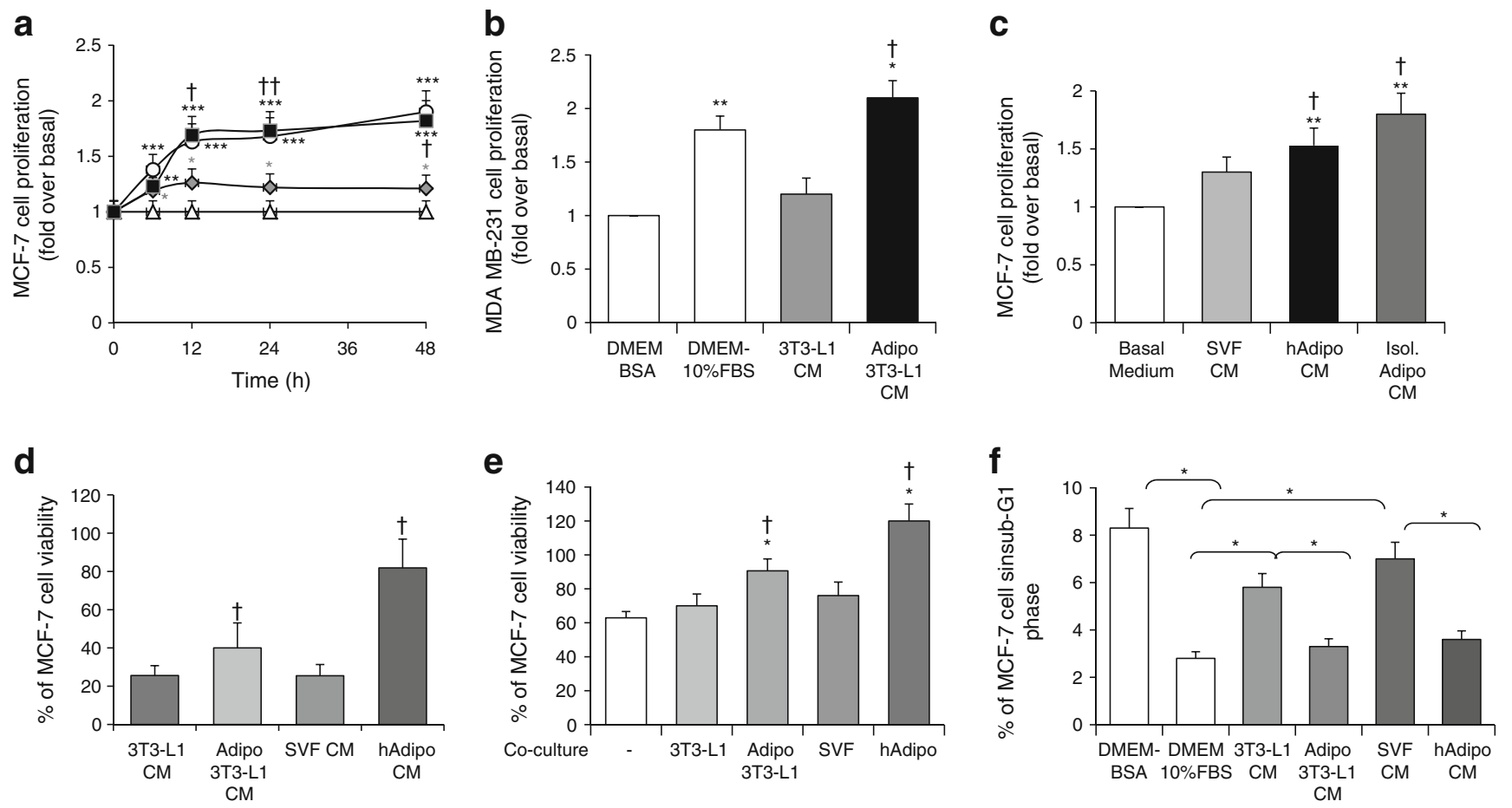

Fig. 1 Effect of adipocyte-released factors on breast cancer cell growth. (a) Undifferentiated mouse 3T3-L1 cells or mature 3T3-L1 adipocytes were incubated with serum-free DMEM for $8 \mathrm{~h}$. Media were collected from undifferentiated 3T3-L1 cells and from mature 3T3-L1 adipocytes (respectively, 3T3-L1 CM [grey diamonds] and Adipo 3T3-L1 CM [black squares]) and added to serum-starved MCF-7 breast cancer cells for $6,12,24$ and $48 \mathrm{~h}$. As a control, MCF-7 cells were also incubated with DMEM without serum supplementation (DMEM-BSA [white triangles]) or with 10\% FBS (DMEM $10 \%$ FBS [white circles]). The cells were then counted as described in the Methods and the results reported as fold increase over basal (cell count in DMEM-BSA). (b) 3T3-L1 CM and Adipo 3T3-L1 CM were added to serum-starved MDA-MB-231 breast cancer cells for $24 \mathrm{~h}$. As a control, MDA-MB-231 cells were also incubated with DMEM-BSA or with DMEM $10 \%$ FBS. Cells were then counted as described in the Methods and the results reported as fold increase over basal (cell count in DMEM-BSA). (c) Adipose-derived SVF, differentiated adipocytes (hAdipo) or mature adipocytes (Isol. Adipo) isolated from breast surgery $(n=12)$ were incubated with serum-free medium for $8 \mathrm{~h}$. Media were collected (CM) and added to serum-starved MCF-7 cells for $24 \mathrm{~h}$. As a control, MCF-7 cells were also incubated with medium without serum supplementation (basal medium). Cells were then counted and the results reported as fold increase over basal (cell count in basal medium). (d) 3T3-L1 CM, Adipo 3T3-L1 CM, SVF CM and hAdipo $\mathrm{CM}$ were added to serum-starved MCF-7 for $48 \mathrm{~h}$. Cell viability was then assessed by sulforhodamine assay as described in the Methods and the results reported as percentage of viable cells compared with cells in DMEM 10\% FBS, considered as $100 \%$ viable cells. (e) MCF-7 cells were seeded in the upper chamber of a transwell culture system with or without 3T3-L1, Adipo 3T3-L1, SVF or hAdipo in the lower chamber and cell viability was determined by crystal violet staining as described in the Methods. The results are reported as percentage of stained cells. ${ }^{*} p<0.05, * * p<0.01, * * * p<0.001$ compared with basal; ${ }^{\dagger} p<0.05$; ${ }^{\dagger \dagger} p<0.01$ compared with $3 \mathrm{~T} 3-\mathrm{L} 1$ or SVF CM. (f) MCF-7 cells were starved in serum-free DMEM for $18 \mathrm{~h}$ and incubated with 3T3-L1 CM, Adipo 3T3-L1 CM, SVF CM and hAdipo CM for $24 \mathrm{~h}$. Cells were stained with propidium iodide and the ratio of cells in subG1 phase was determined by cytometric analysis, as described in the Methods. ${ }^{*} p<0.05$, comparing indicated data For all the panels in the figure, data in the graphs represent the mean $\pm \mathrm{SD}$ of at least four independent triplicate experiments 
cell viability was increased by 3T3-L1 and human mammary adipocyte CM by 1.6 and 2.6 fold, respectively compared with their undifferentiated precursors (Fig. 1d).

Again, when co-cultured with 3T3-L1 and human adipocytes, MCF-7 displayed higher viability, comparable with that observed for cells cultured in DMEM-10\% FBS. A much lower effect was observed when MCF-7 cells were co-cultured with either 3T3-L1 fibroblasts or SVF undifferentiated cells (Fig. 1e).

To further investigate whether growth-promoting action was dependent on oestrogen release by mature adipocytes, MCF-7 cells were incubated with mammary adipocyte $\mathrm{CM}$ in the presence of $1 \mu \mathrm{mol} / 1$ Tamoxifen, a selective oestrogen receptor antagonist (ESM Fig. 1). No significant effect was elicited by tamoxifen on the adipocyte growth-promoting action. Also, exposure of the cells to anti-leptin antibodies did not change the effect of adipocyte $\mathrm{CM}$ on cancer cell growth (ESM Fig. 1).

Moreover, incubation of MCF-7 cells with $\mathrm{CM}$ from 3T3-L1 fibroblasts only slightly decreased the number of cell deaths induced by growth factor deprivation, reducing the number of cells in sub-G1 phase by $25 \%$ (Fig. 1f). Interestingly, cell death was substantially rescued by incubation with CM from 3T3-L1 adipocytes, at levels similar to those achieved with $10 \%$ FBS medium (Fig. 1f). Again, the number of cell deaths was decreased following incubation with CM from mammary adipocytes, and from undifferentiated SVF cells (Fig. 1f), although the effect of the latter $\mathrm{CM}$ was lower.

Adipocyte factors induce MAPK, PIBK and JAK/STAT activation in MCF-7 cells To gain further insight into the molecular mechanism by which adipocytes promote breast cancer cell growth, we tested the ability of adipocyte conditioned media to induce mitogen-activated protein kinase (MAPK), phosphatidylinositol 3-kinase (PI3K) and JAK/ STAT activation. To this aim, MCF-7 cells were treated with CM from 3T3-L1 or from mammary adipocytes for $12 \mathrm{~h}$ (ESM Fig. 2). Western blot and phosphoprotein assay revealed that CM induced the phosphorylation of ERK, Akt and STAT3 (ESM Fig. 2).

Release of cytokines/chemokines and growth factors by 3T3L1 and human mammary adipocytes Based on the observed induction of signalling pathways, we have screened for the ability of 3T3-L1 and mammary adipocytes to release cytokines/chemokines and growth factors. Both 3T3-L1 fibroblasts and undifferentiated SVF cells released detectable amounts of IL-4, IL-6, IL-10, IFN $\gamma, \mathrm{KC} / \mathrm{IL}-8, \mathrm{MIP}-1 \beta$, RANTES and TNF- $\alpha$; MIP- $1 \alpha$ was exclusively detected in the medium of 3T3-L1 pre-adipocytes and not of human SVF cells. However, the levels of KC/IL-8 and RANTES were higher both in 3T3-L1 and human adipocytes, compared with their respective precursors. Moreover, increased levels of IL- 6 and MIP- $1 \beta$ were detected in the $\mathrm{CM}$ of human adipocytes while not in the CM of 3T3-L1 adipocytes.

We also screened for growth factor release and found that VEGF, bFGF, PDGF and IGF-1 were detectable in both preadipocytes and adipocytes. However, while there was no significant difference in the content of PDGF and bFGF, the amount of VEGF was significantly reduced in the $\mathrm{CM}$ of both 3T3-L1 and human mammary adipocytes. At variance, IGF-1 was about fivefold higher in the CM of differentiated 3T3-L1 and mammary adipocytes (Table 1).

Similar to protein levels in the conditioned media, $K c$ (also known as Cxcl1)/IL-8, RANTES and IGF-1 mRNA levels were higher by 5,6 and 6.5 fold, and 4, 4.5 and 7.5 fold, respectively, in differentiated 3T3-L1 cells and in human adipocytes, compared with their undifferentiated counterparts (Fig. 2).

Glucose and fatty acids modify adipocyte growth-promoting action Next, 3T3-L1 adipocytes were cultured for $24 \mathrm{~h}$ either in $5.5 \mathrm{mmol} / 1$ glucose (LG, low glucose), a concentration corresponding to normal fasting glucose levels in humans, or in $25 \mathrm{mmol} / \mathrm{l}$ glucose ( $\mathrm{HG}$, high glucose), corresponding to the regular culture condition for this cell line, but resembling hyperglycaemia in humans. Moreover, we added to culture media either $0.5 \mu \mathrm{mol} / 1$ oleate or $10 \mu \mathrm{mol} / 1$ palmitate, two major fatty acids whose plasma levels are increased in dysmetabolic conditions. Media were changed and cells were allowed to secrete factors into freshly added serum-free medium. After $8 \mathrm{~h}$, media were collected and tested for the content of cytokines/chemokines and growth factors. No significant change was detected for IL-4, IL-6, IL-10, IFN- $\gamma$, MIP- $1 \alpha$, MIP- $1 \beta$, TNF- $\alpha$, PDGF, bFGF and VEGF (data not shown). However, 3T3-L1 adipocytes cultured in $\mathrm{HG}$ medium released significantly higher levels of KC (Fig. 3a), RANTES (Fig. 3b) and IGF-1 (Fig. 3c), compared with those cultured in LG medium. Very similar results were obtained for IL-8 (Fig. 3d), RANTES (Fig. 3e) and IGF-1 (Fig. 3f) in differentiated mammary adipocytes. Interestingly, culturing 3T3-L1 and human adipocytes with either oleate or palmitate in LG medium was accompanied by a significant increase of $\mathrm{KC} /$ IL-8, RANTES and IGF-1 (Fig. 3a-f).

We next addressed whether regulation of those adipocyte-produced factors occurred at the transcriptional level. $K c$ and $I g f-1$ mRNA levels were about twofold higher in 3T3-L1 adipocytes cultured in HG medium compared with LG medium (Fig. 4). Similarly, in differentiated human adipocytes, both IL-8 and IGF-1 levels were higher in HG medium than in LG medium. At variance, RANTES (also known as CCL5) mRNA levels were comparable in HG and LG media, both in 3T3-L1 and human adipocytes. 
Table 1 Adipocyte-released cytokines and growth factors

\begin{tabular}{lllll}
\hline Cytokine/growth factor & 3T3-L1 CM (pg/ml) & Adipo 3T3-L1 CM (pg/ml) & SVF CM (pg/ml) & hAdipo CM (pg/ml) \\
\hline IL-2 & ND & ND & ND & ND \\
IL-4 & $0.7 \pm 0.008$ & $0.10 \pm 0.02$ & $1.12 \pm 0.66$ & $1.36 \pm 0.66$ \\
IL-6 & $23.6 \pm 1.13$ & $22.37 \pm 3.47$ & $2,705.58 \pm 541$ & $5,005.06 \pm 1,200^{\dagger}$ \\
IL-10 & $3.70 \pm 0.86$ & $15 \pm 2.6^{*}$ & $10.47 \pm 2.24$ & $6.53 \pm 1.66^{\dagger \dagger}$ \\
GM-CSF & ND & ND & ND & ND \\
IFN- $\gamma$ & $3.73 \pm 0.38$ & $5.59 \pm 0.80$ & $46.70 \pm 9.2$ & $41.64 \pm 19.8$ \\
KC/IL-8 & $989.14 \pm 100.62$ & $10,343.72 \pm 1,551.53^{*}$ & $2,975.59 \pm 300$ & $15,647.85 \pm 190.2^{\dagger \dagger}$ \\
MIP-1 $\alpha$ & $226.31 \pm 18,27$ & $280 \pm 22.32$ & ND & ND \\
MIP-1 $\beta$ & $10.33 \pm 1.53$ & $11.4 \pm 2$ & $3.37 \pm 1.02$ & $25.88 \pm 9.01^{\dagger \dagger}$ \\
RANTES & $1,960 \pm 235.2$ & $4,770 \pm 524.7^{*}$ & $12.27 \pm 1.5$ & $52.54 \pm 0.80$ \\
TNF- $\alpha$ & $143.54 \pm 18.66$ & $162.88 \pm 25.92$ & $1.20 \pm 0.90$ & $2.17 \pm 1.10$ \\
PDGF & $\mathrm{ND}$ & $\mathrm{ND}$ & $19.37 \pm 3.87$ & $2.65 \pm 1.32$ \\
bFGF & $200 \pm 18$ & $660.70 \pm 2.67^{*}$ & $526.53 \pm 105.30$ & $25.58 \pm 5.37$ \\
VEGF & $11,479.20 \pm 1,262.71$ & $4,682.46 \pm 607.71^{*}$ & $28.85 \pm 0.50$ & $295.58 \pm 65.02^{\dagger \dagger}$ \\
IGF-1 & $79.37 \pm 0.88$ & $334.37 \pm 40.41^{*}$ & & $136.66 \pm 5.7^{\dagger \dagger}$ \\
\hline
\end{tabular}

Data represent the mean $\pm \mathrm{SD}$ of at least four independent triplicate experiments. Supernatants from mouse 3T3-L1 pre-adipocytes (3T3-L1 CM), 3T3-L1 adipocytes (Adipo 3T3-L1 CM), human SVF (SVF CM) and differentiated adipocytes (hAdipo CM were collected for $8 \mathrm{~h}$ and tested by using the Bio-Plex multiplex cytokine assay kit and the Bio-Plex multiplex growth factor assay kit as described in the Methods

${ }^{*} p<0.05$ for 3T3-L1 adipocytes vs $3 \mathrm{~T} 3-\mathrm{L} 1 \mathrm{CM} ;{ }^{\dagger} p<0.05,{ }^{\dagger \dagger} p<0.01,{ }^{\dagger \dagger} p<0.001$ for human adipocytes vs SVF CM

However, when oleate or palmitate were added to $L G$ medium, significant increases of RANTES and IGF-1 (also known as IGF1) mRNA levels, while not of $K c / I L-8$, were observed both in 3T3-L1 and human adipocytes.

Thus, these data suggest that glucose may upregulate $\mathrm{KCl}$ $I L-8$ and $I G F-1$, while fatty acids may induce RANTES and $I G F-1$ at mRNA levels.

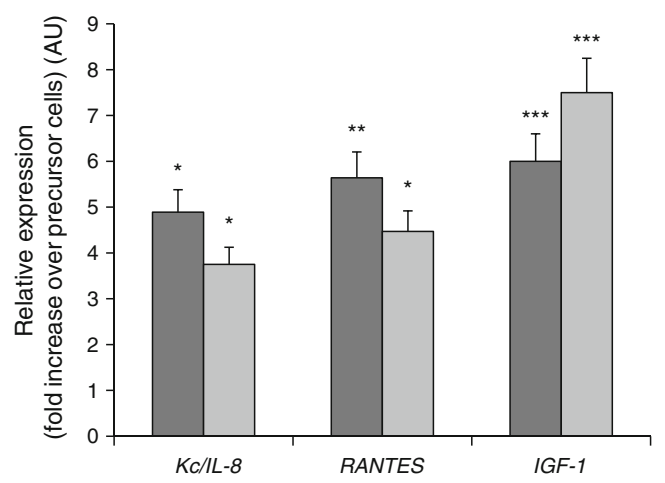

Fig. $2 \mathrm{Kc} / \mathrm{IL}-8, \mathrm{RANTES}$ and $I G F-1$ expression in differentiated adipocytes vs undifferentiated precursors. Undifferentiated 3T3-L1 and SVF cells or mature 3T3-L1 and human adipocytes were tested for the levels of mouse $K c$, Rantes and $I g f-1$ mRNA, or of human $I L-8$, RANTES and IGF-1 mRNA by real-time RT-PCR analysis, as described in the Methods. Dark grey columns represent 3T3-L1 adipocytes; light grey columns represent human adipocytes. Bars represent the mean $\pm \mathrm{SD}$ of four independent experiments and show the mRNA levels in these cells relative to those in 3T3-L1/human adipocyte precursor cells. ${ }^{*} p<0.05, * * p<0.01, * * * p<0.001$, in comparison with the respective measurement in precursor cells
Therefore, we tested whether glucose and fatty acids may change the growth-promoting action of murine and human adipocytes. To this end, MCF-7 cells were incubated for $24 \mathrm{~h}$ in the presence of either LG or HG conditioned media from 3T3-L1 and human mammary adipocytes. Preincubation of adipocytes (both 3T3-L1 and human) with HG medium enhanced, by about twofold, their ability to induce MCF-7 cell growth, compared with LG medium (Fig. 5). Moreover, the presence of palmitate or oleate in the LG medium (from both 3T3-L1 and human adipocytes) significantly increased breast cancer cell growth (Fig. 5).

Induction of breast cancer cell growth by adipocytes from lean and obese individuals SVF cells were isolated from abdominal liposuctions carried out in lean $(n=10)$ and obese $(n=10)$ individuals. These cells were differentiated into mature adipocytes and conditioned media were collected as previously described (see Methods). As for mammaryderived specimens (see Fig. 1c), abdominal subcutaneous differentiated adipocytes induced MCF-7 cell growth to a larger extent, compared with undifferentiated SVF cells (Fig. 6) from both lean and obese individuals. However, CM from both undifferentiated SVF cells and differentiated adipocytes obtained from obese individuals were significantly more effective than those obtained from lean individuals in inducing MCF-7 cell growth.

Again, as for mammary adipocytes, the release of IL-8, RANTES and IGF-1 was more abundant in mature subcutaneous adipocytes, compared with their undifferentiated 

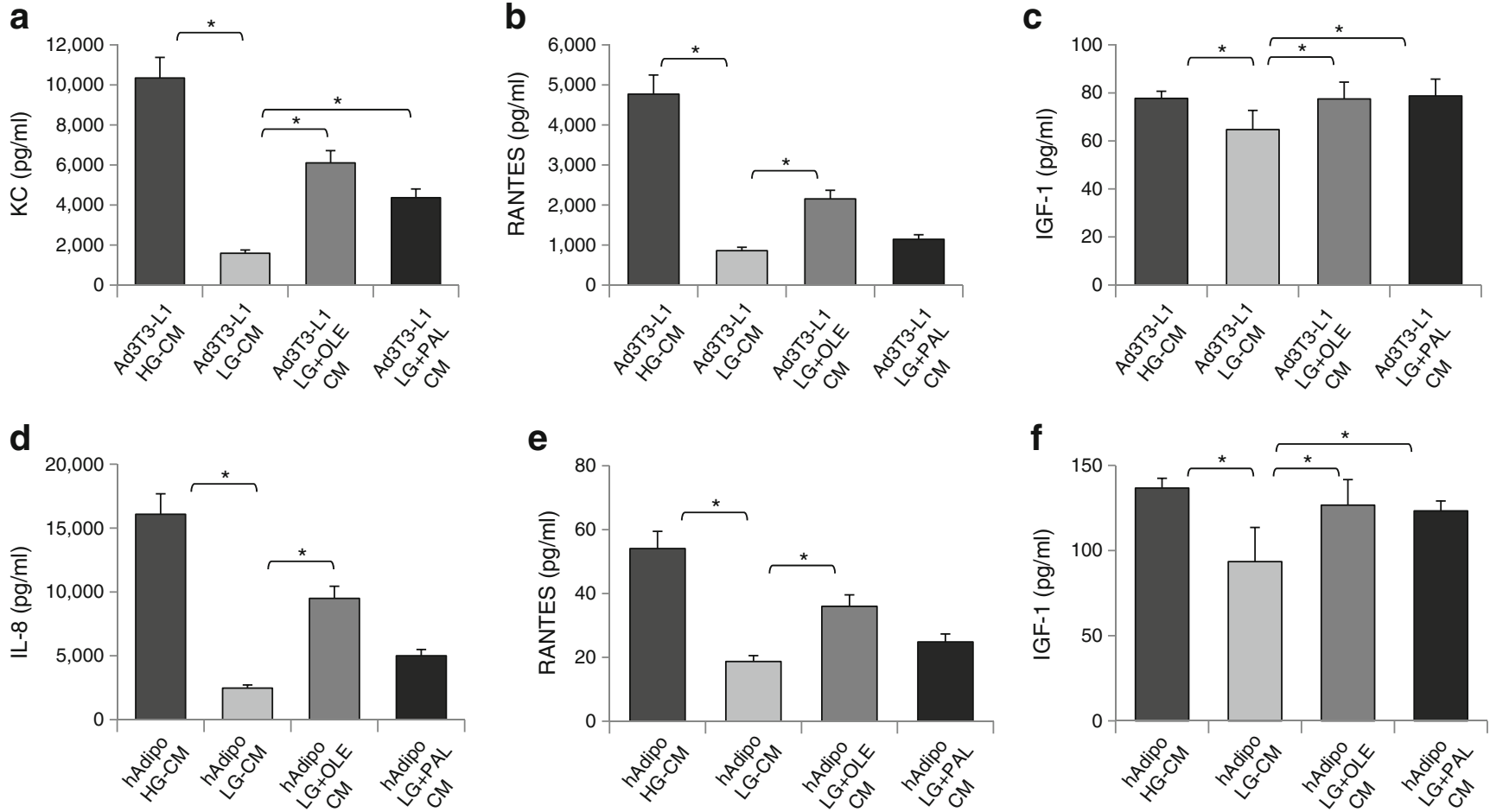

Fig. 3 Effect of glucose and fatty acids on adipocyte-released cytokines and growth factors. Mouse 3T3-L1 adipocytes $(\mathbf{a}-\mathbf{c})$ and human adipocytes $(\mathbf{d}-\mathbf{f})$ were pre-incubated with high glucose DMEM (25 mmol/l glucose, HG) or with low glucose DMEM (5.5 mmol/1 glucose, $\mathrm{LG}$ ) for $24 \mathrm{~h}$ in the presence or absence of $10 \mu \mathrm{mol} / \mathrm{l}$ palmitate (PAL) or $0.5 \mu \mathrm{mol} / \mathrm{l}$ oleate (OLE) for $18 \mathrm{~h}$. They were then further incubated with serum-free DMEM for $8 \mathrm{~h}$. Media were collected (CM)

counterparts (Table 2), both for lean and obese individuals. No difference in IL-4, IL-8, IL-10, IFN- $\gamma$, MIP- $1 \alpha$, MIP$1 \beta$, RANTES, TNF- $\alpha$, PDGF, bFGF and VEGF content was found in the media of SVF cells and mature adipocytes from lean and obese individuals. Nevertheless, IL-6 and IGF-1 were significantly more abundant in CM of obese compared with lean adipocytes (Table 2). Moreover, only IGF-1 levels were higher in SVF cells from obese individuals than in those from lean individuals.

Effect of IGF-1 pathway inhibition on cell growth To address the biological relevance of IGF-1 as an adipocytederived growth-promoting factor, MCF-7 cells were treated with $\mathrm{HG} \mathrm{CM}$ in the presence of $10 \mu \mathrm{mol} / 1 \mathrm{AG1024}$, a specific inhibitor of IGF-1R kinase activity.

AG1024 almost completely prevented the effect of 3T3L1 CM on MCF-7 cell growth (Fig. 7a). Very similar results were obtained for the prevention of the effect induced by mammary (Fig. 7b) as well as subcutaneous abdominal adipocytes, both from lean and from obese individuals (Fig. 7c). Indeed, the induction of MCF-7 cell growth by lean and obese adipocytes was reduced at comparable levels, following treatment with AG1024 (Fig. 7c). Moreover, the increased growth induced by SVF cells from obese and tested by using the Bio-Plex multiplex cytokine assay kit and the Bio-Plex multiplex growth factor assay kit. Values \pm SD for mouse KC (a), RANTES (b) and IGF-1 (c), and for human IL-8 (d), RANTES (e) and IGF-1 (f) are reported in the bar graph $* p<0.05$, comparing indicated data and represent the mean $\pm \mathrm{SD}$ of at least four independent triplicate experiments

individuals was fully rescued by AG1024 (Fig. 7c) and returned to levels similar to those achieved by SVF cells from lean individuals. Similarly, conditioned media from obese adipocytes induced IGF-1R phosphorylation twofold more effectively than those from lean individuals (Fig. 7d). Also, only SVF cells from obese, and not from lean, individuals were able to increase IGF-1R phosphorylation. Finally, these effects were completely blocked by AG1024, indicating a major involvement of IGF-1 (Fig. 7d).

\section{Discussion}

Convincing evidence links obesity and diabetes to the risk of several forms of cancer $[4,11,14]$. We now describe that differentiated adipocytes, both of mouse and of human origin, have a significant impact in enhancing breast cancer cell survival and proliferation. Consistent with previous reports [16], the effect of adipocytes is larger than that of their precursor cells. Interestingly, however, their growthpromoting action is paralleled by a specific secretory pattern of inflammatory cytokines, chemokines and growth factors, which is also affected by culture conditions. In particular, both glucose and fatty acids enhanced the ability of 

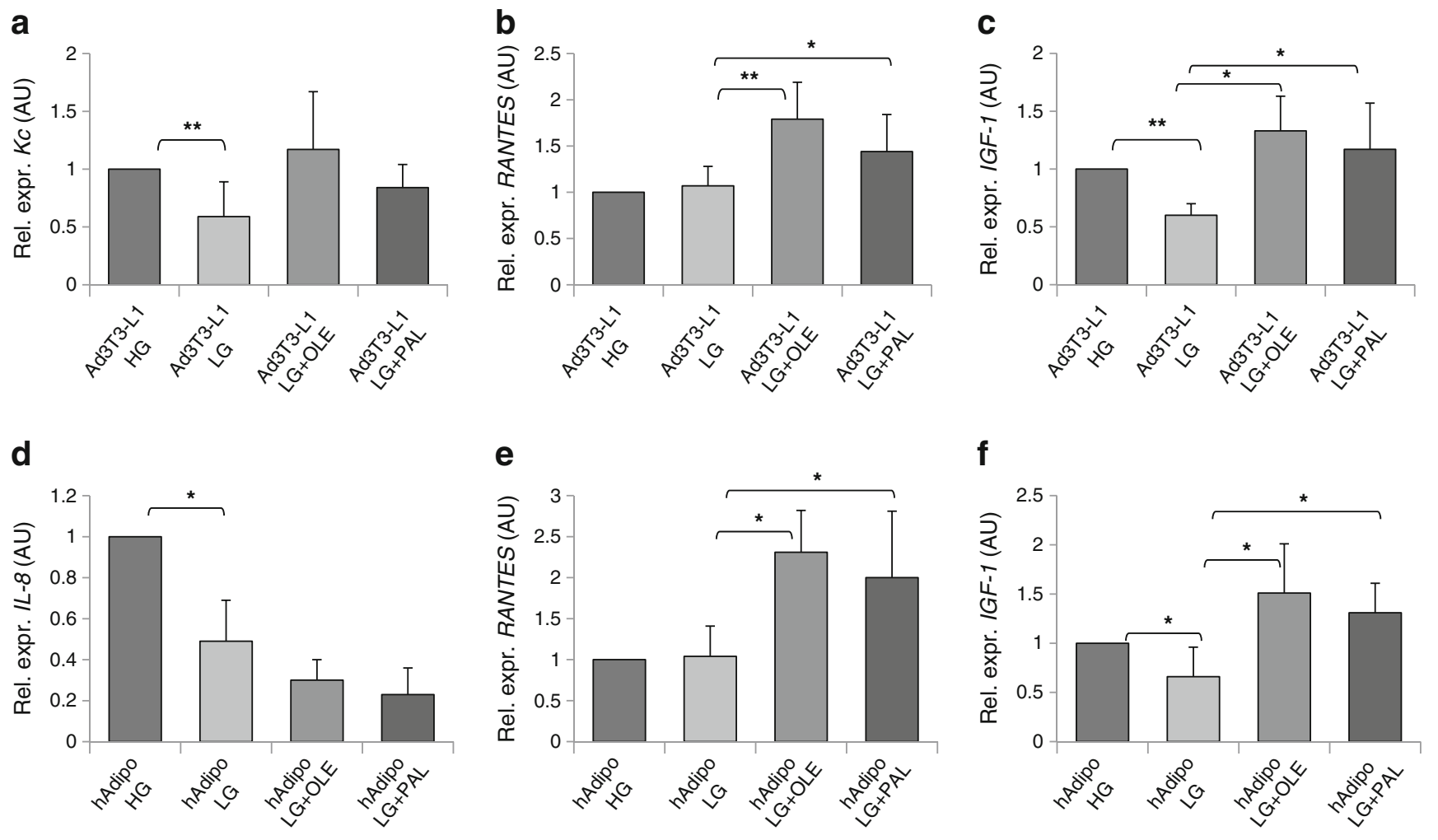

Fig. 4 Glucose and fatty acids control $K c / I L-8$, RANTES and IGF-1 expression. Mouse 3T3-L1 adipocytes (a-c) and human adipocytes (hAdipo) $(\mathbf{d}-\mathbf{f})$ were pre-incubated with either HG DMEM or with LG DMEM for $24 \mathrm{~h}$ in the presence or absence of $10 \mu \mathrm{mol} / 1$ palmitate (PAL) or $0.5 \mu \mathrm{mol} / 1$ oleate (OLE) for $18 \mathrm{~h}$. Following this, mRNA levels of mouse $K c$ (a), Rantes (b) and Igf- 1 (c), and of human $I L-8(\mathbf{d})$,

adipocytes to induce the growth of breast cancer cells. When cultured in $25 \mathrm{mmol} / \mathrm{l}$ glucose (HG), a condition mimicking hyperglycaemia, both 3T3-L1 and human breast mammary adipocytes were more effective in inducing growth of MCF-7 and MDA-MB-231 cells, compared with when they were cultured in $5.5 \mathrm{mmol} / \mathrm{l}$ glucose (LG), which represents normal glycaemia in humans.

Incubation of adipocytes with fatty acids also led to increased growth-promoting action, suggesting that metabolic perturbations may change their secretory pattern. This effect was observed with palmitate or oleate, either individually or in combination (data not shown). Higher concentrations of fatty acids (up to $100 \mu \mathrm{mol} / \mathrm{l}$ palmitate and $10 \mu \mathrm{mol} / \mathrm{l}$ oleate) had a slightly higher effect on both cancer cell growth and secretory profile (data not shown). Moreover, it is known that high concentrations of fatty acids impair insulin sensitivity [28] and this may further affect adipocyte functions.

It has been reported that adipocyte-derived molecules can affect cancer cell growth. For instance, leptin has been shown to induce proliferation of breast cancer cells [29]. However, we found that glucose and fatty acids had no effect on leptin release by mammary adipocytes (data not shown). Nevertheless, treatment of MCF-7 cells with anti-
RANTES (e) and IGF-1 (f) were determined by real-time RT-PCR analysis. Data were normalized on $\beta$-actin as internal standard. Bars represent the mean $\pm \mathrm{SD}$ of four independent experiments and show the mRNA levels in these cells relative to those in 3T3-L1/hAdipo HG cells, reported as 1 in the first column of each bar graph. ${ }^{*} p<0.05$, ${ }^{* *} p<0.01$, comparing indicated data. Rel. expr., relative expression

leptin blocking antibodies did not rescue the effect of $\mathrm{HG}$ adipocyte CM on cell growth (ESM Fig. 1). Adipocytederived oestrogens have also been related to breast cancer development and progression [30]. It seems unlikely that oestrogens could mediate the effect of glucose and fatty acids, however, since adipocyte $\mathrm{CM}$ elicited a similar effect in the oestrogen-independent MDA-MB-231 cells and it was unchanged following tamoxifen treatment of MCF-7 cells. We therefore tested whether glucose and fatty acids could modify the release of cytokines, chemokines and growth factors by adipocytes. The secretory patterns obtained by mouse 3T3-L1 and human mammary adipocytes were somewhat different. For example, MIP- $1 \alpha$ and TNF- $\alpha$ were more abundant in 3T3-L1 cells, while IL-6 and IFN- $\gamma$ were more abundant in human cells. However, IL-8 and RANTES were released to a larger extent by both differentiated 3T3-L1 cells and human adipocytes, compared with their precursors, indicating that common changes were present in the differentiation process. The increased release of IL-8 and RANTES was paralleled by a similarly sized increase in their mRNA levels.

Interestingly, both glucose and fatty acids increased the release of IL-8 and RANTES, suggesting that the metabolic 

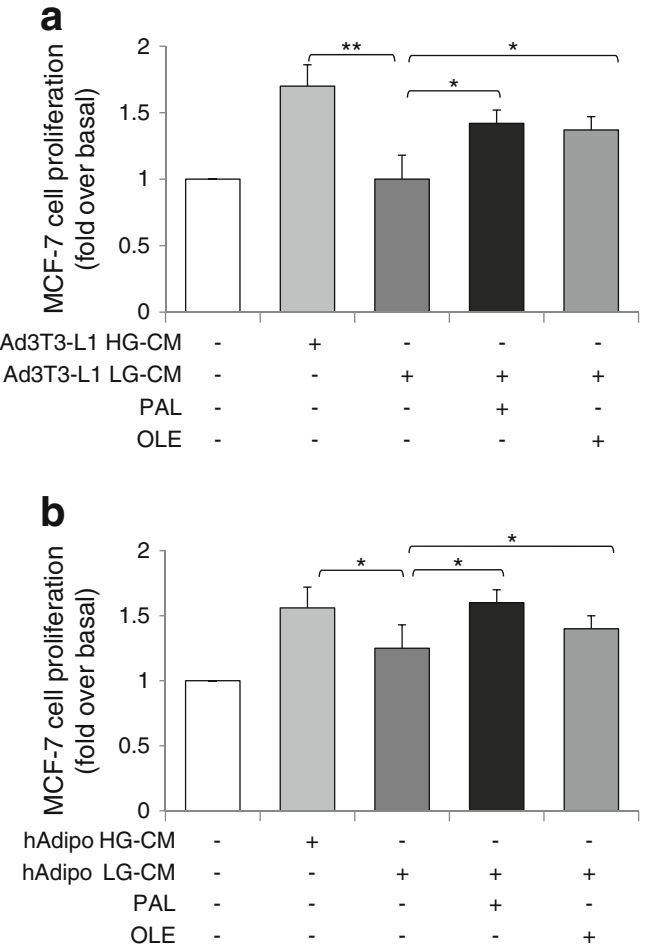

Fig. 5 Effect of glucose and fatty acids on adipocyte growthpromoting action. Mouse 3T3-L1 adipocytes (a) and human adipocytes (b) were pre-incubated with either HG DMEM or with LG DMEM for $24 \mathrm{~h}$ in the presence or absence of $10 \mu \mathrm{mol} / 1$ palmitate or $0.5 \mu \mathrm{mol} / 1$ oleate for $18 \mathrm{~h}$. They were then further incubated with serum-free DMEM for $8 \mathrm{~h}$. Media were collected (CM) and added to serum-starved MCF-7 cells for $24 \mathrm{~h}$. Cells were then counted and the results reported as fold increase over basal (cell count in DMEM-BSA). ${ }^{*} p<0.05,{ }^{* *} p<0.01$, comparing indicated data. Data in the bar graphs represent the mean $\pm \mathrm{SD}$ of at least four independent triplicate experiments

environment could further modify adipocyte releasing properties. Similar results were also found for IGF-1, which was more abundant in the medium of mature adipocytes and upregulated by glucose and fatty acids. bFGF also tended to be detected in higher amounts in the mature adipocytes, while VEGF levels were more elevated in the undifferentiated precursors. However, neither bFGF nor VEGF were significantly regulated by nutrients.

We have obtained evidence that glucose and fatty acids may regulate IL-8, RANTES and IGF-1 at different levels. Culturing adipocytes in $\mathrm{HG}$ is accompanied by a significant upregulation of $I L-8$ and $I G F-1$, while not of RANTES mRNA, compared with low glucose. At variance, both oleate and palmitate enhance IGF-1 and RANTES, while not $I L-8$ mRNA levels. Altogether, these data raise the possibility that, beside regulating gene expression, nutrients may control adipocyte secretion process.

Thus, these findings are consistent with the hypothesis that adipocytes, in an appropriate metabolic environment, may contribute to tumour growth and progression by releasing factors that may function to promote cancer cell proliferation. In particular, RANTES, IL-8 and IGF-1 represent good candidates since: (1) they are produced in the adipocytes and released in the culture medium; (2) their production/release is induced by nutritional factors, such as glucose and fatty acids; and (3) they have been implicated in the control of cancer cell phenotypes. For instance, RANTES and IL-8 are elevated in plasma of obese individuals [31-33] and have been largely implicated in obesityrelated health complications [34-36] as well as in the mechanisms of cancer progression [37-39].

IGF-1 also plays a pivotal role in the progression of many forms of cancer, including breast cancer [40-42]. Indeed IGF-1 is involved in the development of normal mammary gland and in the onset and/or progression of mammary tumours [42], as well as in the development of drug resistance [43].

It was recently reported that, in pancreatic cancer, autocrine production of IGF-1 by cancer cells enhances the production of RANTES by stromal cells, thereby providing a paracrine loop to facilitate tumour progression [44]. Moreover, expression profile studies indicate that IL-8 and IGF-1 are produced at higher levels in tissues from patients with high-grade breast cancer than in tissues from patients with low-grade breast cancer [45].

We have also found that adipocytes obtained from subcutaneous fat specimens from obese individuals were capable of producing larger amounts of IGF-1 compared with lean controls. This is consistent with higher circulating IGF1 levels often found in obesity [46]. Surprisingly, the difference was also significant when IGF-1 was measured in the conditioned media of undifferentiated SVF cells from obese individuals. Thus, IGF-1 upregulation appears to occur early

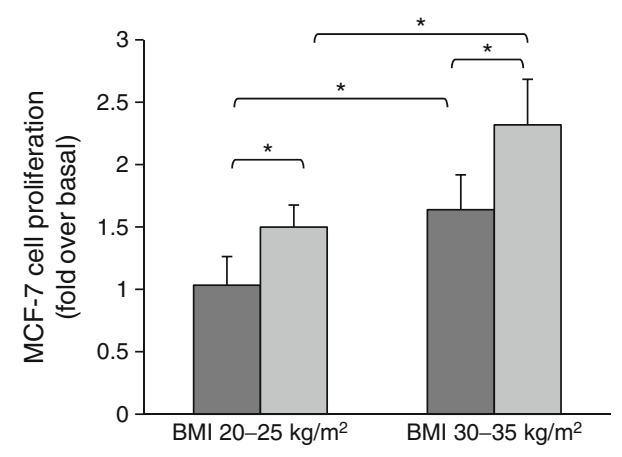

Fig. 6 Cancer cell growth induced by adipocytes from lean and obese individuals. SVF cells and human adipocytes isolated from obese (BMI 30-35) and lean women (BMI 20-25) were incubated with serum-free medium for $8 \mathrm{~h}$. Media were collected (CM) and added to serumstarved MCF-7 cells for $24 \mathrm{~h}$. Then, cells were counted and the results reported as fold increase over basal (cell count in medium without serum supplementation). Dark grey columns represent SVF CM; light grey columns represent human adipocyte (hAdipo) CM. ${ }^{*} p<0.05$, comparing indicated data. Data in the bar graphs represent the mean $\pm \mathrm{SD}$ of at least four independent triplicate experiments 
Table 2 Cytokines and growth factors released by adipocytes from lean and obese individuals

\begin{tabular}{|c|c|c|c|c|}
\hline \multirow[t]{2}{*}{ Cytokine/growth factor } & \multicolumn{2}{|l|}{ BMI 21-25 } & \multicolumn{2}{|l|}{ BMI 30-35 } \\
\hline & SVF CM (pg/ml) & hAdipo CM (pg/ml) & SVF CM (pg/ml) & hAdipo CM (pg/ml) \\
\hline IL-2 & ND & ND & ND & ND \\
\hline IL-4 & $1.09 \pm 0.60$ & $1.49 \pm 0.53$ & $0.79 \pm 0.06$ & $0.96 \pm 0.006$ \\
\hline IL-6 & $2,446.87 \pm 489.37$ & $6,420.31 \pm 1,348.26^{*}$ & $2,180.64 \pm 436.12$ & $12,367.95 \pm 2,597.26^{* \dagger}$ \\
\hline IL-10 & $11.40 \pm 2.66$ & $6.75 \pm 1.48^{*}$ & $9.61 \pm 2.32$ & $5.90 \pm 1.99 *$ \\
\hline GM-CSF & ND & ND & ND & ND \\
\hline IFN- $\gamma$ & $45.82 \pm 9.62$ & $46.7 \pm 10.27$ & $38.86 \pm 12.72$ & $41.64 \pm 18.80$ \\
\hline IL-8 & $2,556.45 \pm 350.45$ & $25,019.32 \pm 1,264.48 * *$ & $1,229.32 \pm 380.12$ & $22,271.66 \pm 5,308.75 * *$ \\
\hline MIP-1 $\alpha$ & $58.82 \pm 7.98$ & $136.23 \pm 9.62$ & $54.14 \pm 4.80$ & $52.95 \pm 7.83$ \\
\hline MIP- $1 \beta$ & $10.33 \pm 3.46$ & $27.96 \pm 3.27 *$ & $3.52 \pm 0.58$ & $34.62 \pm 8.44^{*}$ \\
\hline RANTES & $17.98 \pm 3.40$ & $73.65 \pm 9.68^{*}$ & $12.45 \pm 2.27$ & $45.26 \pm 11.35^{* *}$ \\
\hline TNF- $\alpha$ & $204.08 \pm 15.35$ & $291.65 \pm 35.82$ & $173.49 \pm 18.52$ & $200.04 \pm 21.71$ \\
\hline PDGF & $2.48 \pm 1.35$ & $3.04 \pm 0.88$ & $2.80 \pm 0.52$ & $3.16 \pm 0.71$ \\
\hline bFGF & $28.63 \pm 11.90$ & $36.88 \pm 12.66$ & $14.68 \pm 3.74$ & $24.55 \pm 6.27$ \\
\hline VEGF & $620.53 \pm 165.61$ & $178.54 \pm 85.01 * *$ & $483.06 \pm 29.48$ & $322.20 \pm 71.55$ \\
\hline IGF-1 & $115 \pm 54.46$ & $666 \pm 40.42 *$ & $193.33 \pm 64.29^{\dagger}$ & $1,073.33 \pm 22.30 * * \dagger$ \\
\hline
\end{tabular}

SVF cells and differentiated SVF (hAdipo) isolated from obese (BMI 30-35; $n=10$ ) and lean women (BMI 20-25; $n=10$ ) were incubated with serum-free medium for $8 \mathrm{~h}$. Media were collected $(\mathrm{CM})$ and analysed by using the Bio-Plex multiplex human cytokine assay kit and the Bio-Plex multiplex human growth factor assay kit. Determinations were done in triplicate

${ }^{*} p<0.05,{ }^{*} p<0.01$ for adipocytes vs SVF CM within the same group; ${ }^{\dagger} p<0.05 ;{ }^{\dagger \dagger} p<0.01$ for SVF or hAdipo CM of one group vs the other ND, no data

during adipocyte differentiation, is enhanced in obesity and is maintained in cultured mesenchymal stem cells. This could possibly be due to epigenetic changes in IGF-1 gene expression regulation [47]. Interestingly, however, upregulation was only observed for $I G F-1$ and not for other chemokines and growth factors, both in undifferentiated SVF and in mature adipocytes.
IGF-1 in the adipose tissue may act either as an autocrine factor [48], regulating adipose tissue development and homoeostasis, or in a paracrine manner, regulating survival and function of the surrounding cells, including cancer cells. Inhibition of IGF-1 function, indeed, almost completely abolished the effect of adipocyte-released factors, thereby indicating that IGF-1 is a pivotal factor in adipocyte a

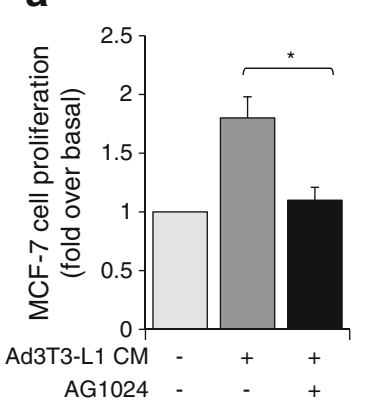

b

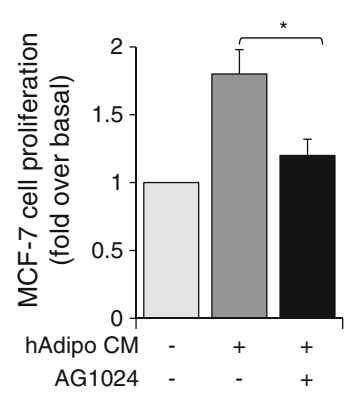

C

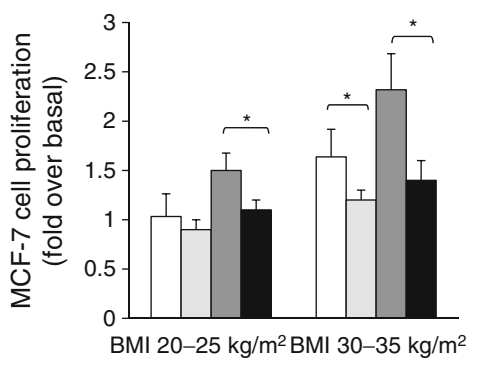

d

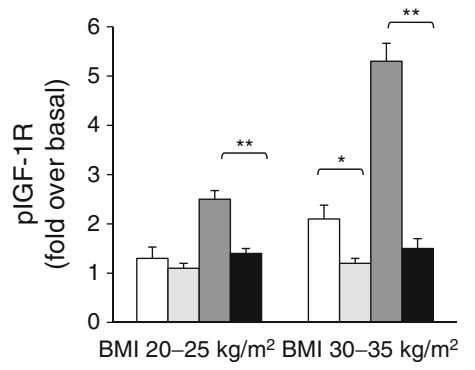

Fig. 7 Effect of IGF-1 pathway inhibition on cell growth. Conditioned media collected from mouse 3T3-L1 adipocytes (a) and mammary adipocytes (b) and subcutaneous abdominal SVF and adipocytes isolated from obese (BMI 30-35) and lean women (BMI 20-25) (c) were added to serum-starved MCF-7 cells for $24 \mathrm{~h}$ in the presence or absence of $10 \mu \mathrm{mol} / 1 \mathrm{AG} 1024$. Cells were then counted and the results reported as fold increase over basal (cell count in serum-free medium). White columns, SVF CM; light grey columns, SVF CM + Ag1024; dark grey columns, human adipocyte (hAdipo) CM; black columns, hAdipo CM + Ag1024. (d) MCF-7 cells were exposed to conditioned media collected from subcutaneous abdominal SVF and adipocytes isolated from obese (BMI 30-35) and lean women (BMI 20-25) for $24 \mathrm{~h}$ and proteins extracted were tested for the presence of phosphorylated IGF-1R by using the Bio-Plex phosphoprotein assay kit as described in Methods. White columns, SVF CM; light grey columns, SVF CM + Ag1024; dark grey columns, hAdipo CM; black columns, hAdipo CM + Ag1024. ${ }^{*} p<0.05,{ }^{*} * p<0.01$, comparing indicated data. Data in the bar graphs represent the mean $\pm \mathrm{SD}$ of at least four independent triplicate experiments 
regulation of cancer cell growth. However, these results are limited to breast cancer models, in which the importance of the local environment, including adipocyte function, has been largely documented. Further studies are needed to assess the relevance of adipose-related factors in the control of other cancer types. Moreover, the communications between cancer cells and adipocytes may be bidirectional. Release of TNF- $\alpha$ from cancer cells may affect adipocyte differentiation and function, possibly increasing NEFA release, to provide additional feed for the cancer cells $[49,50]$.

Thus, we have described that IGF-1 release by adipocytes is enhanced in obesity and is regulated by metabolic perturbations. Other factors, such as RANTES and IL-8, which are not primarily upregulated in obese individuals, are also enhanced by glucose and fatty acids and may as well contribute to adipocyte control of breast cancer cell growth.

Acknowledgements The authors are grateful to S. Libertini (University of Naples, Naples, Italy) and C. Passaro (University of Naples, Naples, Italy) for technical help with cytofluorimetric assays and to G. Perruolo (CNR, Naples, Italy) and G. Portella (University of Naples, Naples, Italy) for helpful discussion and advice.

Funding This study was supported in part by the European Community's FP6 PREPOBEDIA (201681), the European Foundation for the Study of Diabetes (EFSD), the Associazione Italiana per la Ricerca sul Cancro (AIRC) and by the Ministero dell'Università e della Ricerca Scientifica (grants PRIN and FIRB-MERIT).

Duality of interest The authors declare that there is no duality of interest associated with this manuscript.

Contribution statement VD'E was the main contributor in terms of conception, design, acquisition and interpretation of data and in drafting the article. FP, AH, DL, DT, GM, LC and CM mainly contributed in conceptual design and acquisition of data. FB and US mainly contributed in terms of conceptual design, analysis, interpretation and discussion of the results. PF mainly contributed in terms of conceptual design, interpretation and discussion of the results and supervision of the overall work. All the authors critically revised the article and approved the final version.

Open Access This article is distributed under the terms of the Creative Commons Attribution Noncommercial License which permits any noncommercial use, distribution, and reproduction in any medium, provided the original author(s) and the source are credited.

\section{References}

1. La Vecchia C, Giordano SH, Hortobagyi GN, Chabner B (2011) Overweight, obesity, diabetes, and risk of breast cancer: interlocking pieces of the puzzle. Oncologist 16:726-729

2. Khandekar MJ, Cohen P, Spiegelman BM (2011) Molecular mechanisms of cancer development in obesity. Nat Rev Cancer 11:886-895

3. Calle EE, Thun MJ (2004) Obesity and cancer. Oncogene 23:6365-6378
4. Renehan A, Smith U, Kirkman MS (2010) Linking diabetes and cancer: a consensus on complexity. Lancet 375:2201-2202

5. Johnson JA, Carstensen B, Witte D, Bowker SL, Lipscombe L, Renehan AG (2012) Diabetes and cancer (1): evaluating the temporal relationship between type 2 diabetes and cancer incidence. Diabetologia 55:1607-1618

6. Chen J (2011) Multiple signal pathways in obesity-associated cancer. Obes Rev 12:1063-1070

7. Barone BB, Yeh HC, Snyder CF et al (2010) Postoperative mortality in cancer patients with preexisting diabetes: systematic review and meta-analysis. Diabetes Care 33:931-939

8. Renehan AG, Yeh HC, Johnson JA, Wild SH, Gale EA, Møller H (2012) Diabetes and cancer (2): evaluating the impact of diabetes on mortality in patients with cancer. Diabetologia 55: 1619-1632

9. Poulos SP, Hausman DB, Hausman GJ (2010) The development and endocrine functions of adipose tissue. Mol Cell Endocrinol 323:20-23

10. Carswell KA, Lee MJ, Fried SK (2012) Culture of isolated human adipocytes and isolated adipose tissue. Meth Mol Biol 806:203-214

11. Calle EE, Kaaks R (2004) Overweight, obesity and cancer: epidemiological evidence and proposed mechanisms. Nat Rev Cancer 4:579-591

12. Rajala MW, Scherer PE (2003) Minireview: the adipocyte - at the crossroads of energy homeostasis, inflammation, and atherosclerosis. Endocrinology 144:3765-3773

13. Frühbeck G, Gómez-Ambrosi J, Muruzábal FJ, Burrell MA (2001) The adipocyte: a model for integration of endocrine and metabolic signaling in energy metabolism regulation. Am J Physiol Endocrinol Metab 280:E827-E847

14. Park J, Euhus DM, Scherer PE (2011) Paracrine and endocrine effects of adipose tissue on cancer development and progression. Endocr Rev 32:550-570

15. Feldmann M (2008) Many cytokines are very useful therapeutic targets in disease. J Clin Invest 118:3533-3536

16. Iyengar P, Combs TP, Shah SJ et al (2003) Adipocyte-secreted factors synergistically promote mammary tumorigenesis through induction of anti-apoptotic transcriptional programs and protooncogene stabilization. Oncogene 22:6408-6423

17. Macciò A, Madeddu C, Mantovani G (2009) Adipose tissue as target organ in the treatment of hormone-dependent breast cancer: new therapeutic perspectives. Obes Rev 10:660-670

18. Petrelli JM, Calle EE, Rodriguez C, Thun MJ (2002) Body mass index, height, and postmenopausal breast cancer mortality in a prospective cohort of US women. Cancer Causes Contr 13:325-332

19. Larsson SC, Mantzoros CS, Wolk A (2007) Diabetes mellitus and risk of breast cancer: a meta-analysis. Int J Cancer 121:856-862

20. Lipscombe LL, Goodwin PJ, Zinman B, McLaughlin JR, Hux JE (2008) The impact of diabetes on survival following breast cancer. Breast Cancer Res Treat 109:389-395

21. Lorincz AM, Sukumar S (2006) Molecular links between obesity and breast cancer. Endocr Relat Cancer 13:279-292

22. Chavey C, Mari B, Monthouel MN et al (2003) Matrix metalloproteinases are differentially expressed in adipose tissue during obesity and modulate adipocyte differentiation. J Biol Chem 278:11888-11896

23. Isakson P, Hammarstedt A, Gustafson B, Smith U (2009) Impaired preadipocyte differentiation in human abdominal obesity: role of Wnt, tumor necrosis factor-alpha, and inflammation. Diabetes 58:1550-1557

24. Spector AA (1975) Fatty acid binding to plasma albumin. J Lipid Res 16:165-179

25. Skehan P, Storeng R, Scudiero D (1990) New colorimetric cytotoxicity assay for anticancer-drug screening. J Natl Cancer Inst $82: 1107-1112$ 
26. Chiba K, Kawakami K, Tohyama K (1998) Simultaneous evaluation of cell viability by neutral red, MTT and crystal violet staining assays of the same cells. Toxicol Vitro 12:251-258

27. Alberobello AT, D'Esposito V, Marasco D (2010) Selective disruption of insulin-like growth factor-1 (IGF-1) signaling via phosphoinositide-dependent kinase-1 prevents the protective effect of IGF-1 on human cancer cell death. J Biol Chem 285:6563-6572

28. Guilherme A, Virbasius JV, Puri V, Czech MP (2008) Adipocyte dysfunctions linking obesity to insulin resistance and type 2 diabetes. Nat Rev Mol Cell Biol 9:367-377

29. Dieudonne MN, Machinal-Quelin F, Serazin-Leroy V, Leneveu MC, Pecquery R, Giudicelli Y (2002) Leptin mediates a proliferative response in human MCF7 breast cancer cells. Biochem Biophys Res Commun 293:622-628

30. Cleary MP, Grossmann ME (2009) Minireview: obesity and breast cancer: the estrogen connection. Endocrinology 150:2537-2542

31. Wu H, Ghosh S, Perrard XD et al (2007) T cell accumulation and regulated on activation, normal $\mathrm{T}$ cell expressed and secreted upregulation in adipose tissue in obesity. Circulation 115:1029-1038

32. Herder C, Illig T, Baumert J, Müller M et al (2008) RANTES/ CCL5 gene polymorphisms, serum concentrations, and incident type 2 diabetes: results from the MONICA/KORA Augsburg casecohort study, 1984-2002. Eur J Endocrinol 158:R1-R5

33. Straczkowski M, Dzienis-Straczkowska S, Stêpieñ A, Kowalska I, Szelachowska M, Kinalska I (2002) Plasma interleukin-8 concentrations are increased in obese subjects and related to fat mass and tumor necrosis factor-alpha system. J Clin Endocrinol Metab 87:4602-4606

34. Braunersreuther V, Zernecke A, Arnaud C et al (2007) Ccr5 but not Ccrl deficiency reduces development of diet-induced atherosclerosis in mice. Arterioscler Thromb Vasc Biol 27:373-379

35. Veillard NR, Kwak B, Pelli G (2004) Antagonism of RANTES receptors reduces atherosclerotic plaque formation in mice. Circ Res 94:253-261

36. Herder C, Haastert B, Müller-Scholze S et al (2005) Association of systemic chemokine concentrations with impaired glucose tolerance and type 2 diabetes: results from the Cooperative Health Research in the Region of Augsburg Survey S4 (KORA S4). Diabetes 54(Suppl 2):S11-S17

37. Murooka TT, RaHbAr R, Fish EN (2009) CCL5 promotes proliferation of MCF-7 cells through mTOR-dependent mRNA translation. Biochem Biophys Res Commun 387:381-386
38. Raman D, Baugher PJ, Thu YM, Richmond A (2007) Role of chemokines in tumor growth. Cancer Lett 256:137-165

39. Waugh DJ, Wilson C (2008) The interleukin-8 pathway in cancer. Clin Cancer Res 14:6735-6741

40. Clayton PE, Banerjee I, Murray PG, Renehan AG (2011) Growth hormone, the insulin-like growth factor axis, insulin and cancer risk. Nat Rev Endocrinol 7:11-24

41. Hankinson SE, Willett WC, Colditz GA et al (1998) Circulating concentrations of insulin-like growth factor-I and risk of breast cancer. Lancet 351:1393-1396

42. Pollak MN, Schernhammer ES, Hankinson SE (2004) Insulin-like growth factors and neoplasia. Nat Rev Cancer 4:505-518

43. Ryan PD, Goss PE (2008) The emerging role of the insulin-like growth factor pathway as a therapeutic target in cancer. Oncologist 13:16-24

44. Makinoshima H, Dezawa M (2009) Pancreatic cancer cells activate CCL5 expression in mesenchymal stromal cells through the insulin-like growth factor-I pathway. FEBS Lett 583:3697-3703

45. Razmkhah M, Jaberipour M, Hosseini A, Safaei A, Khalatbari B, Ghaderi A (2010) Expression profile of IL-8 and growth factors in breast cancer cells and adipose-derived stem cells (ASCs) isolated from breast carcinoma. Cell Immunol 265:80-85

46. Frystyk J, Skjaerbaek C, Vestbo E, Fisker S, Orskov H (1999) Circulating levels of free insulin-like growth factors in obese subjects: the impact of type 2 diabetes. Diabetes Metab Res Rev 15:314-322

47. Sanchez C, Oskowitz A, Pochampally RR (2009) Epigenetic reprogramming of IGF1 and leptin genes by serum deprivation in multipotential mesenchymal stromal cells. Stem Cell 27:375-382

48. Klöting N, Koch L, Wunderlich T et al (2008) Autocrine IGF-1 action in adipocytes controls systemic IGF-1 concentrations and growth. Diabetes 57:2074-2082

49. Meng L, Zhou J, Sasano H, Suzuki T, Zeitoun KM, Bulun SE (2001) Tumor necrosis factor alpha and interleukin 11 secreted by malignant breast epithelial cells inhibit adipocyte differentiation by selectively down-regulating CCAAT/enhancer binding protein alpha and peroxisome proliferator-activated receptor gamma: mechanism of desmoplastic reaction. Cancer Res 61:2250-2255

50. Guerrero J, Tobar N, Cáceres M et al (2010) Soluble factors derived from tumor mammary cell lines induce a stromal mammary adipose reversion in human and mice adipose cells. Possible role of TGFbeta1 and TNF-alpha. Breast Cancer Res Treat 119:497-508 